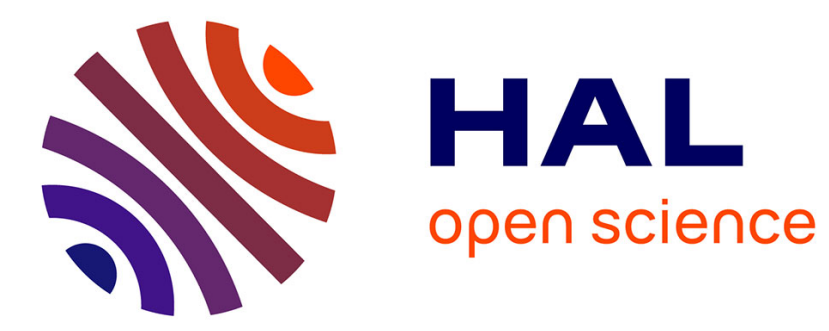

\title{
On normal partitions in cubic graphs
} Jean-Luc Fouquet, Jean-Marie Vanherpe

\section{To cite this version:}

Jean-Luc Fouquet, Jean-Marie Vanherpe. On normal partitions in cubic graphs. Discussiones Mathematicae Graph Theory, 2009, 29 (2), pp.293-312. hal-00325250

\section{HAL Id: hal-00325250 \\ https://hal.science/hal-00325250}

Submitted on 27 Sep 2008

HAL is a multi-disciplinary open access archive for the deposit and dissemination of scientific research documents, whether they are published or not. The documents may come from teaching and research institutions in France or abroad, or from public or private research centers.
L'archive ouverte pluridisciplinaire HAL, est destinée au dépôt et à la diffusion de documents scientifiques de niveau recherche, publiés ou non, émanant des établissements d'enseignement et de recherche français ou étrangers, des laboratoires publics ou privés. 


\title{
On normal partitions in cubic graphs
}

\author{
J.L. Fouquet and J.M. Vanherpe \\ L.I.F.O., Faculté des Sciences, B.P. 6759 \\ Université d'Orléans, 45067 Orléans Cedex 2, FR
}

\begin{abstract}
A normal partition of the edges of a cubic graph is a partition into trails (no repeated edge) such that each vertex is the end vertex of exactly one trail of the partition. We investigate this notion and give some results and problems.
\end{abstract}

Key words: Cubic graph; Edge-partition;

\section{Introduction and notations}

Let $G=(V, E)$ be a cubic graph (loops and multiple edges are allowed) and let $\mathcal{T}=\left\{T_{1}, T_{2} \ldots, T_{k}\right\}$ be a partition of $E(G)$ into trails (no repeated edge). Every vertex $v \in V(G)$ is either an end vertex three times in the partition and we shall say that $v$ is an eccentric vertex, or an end vertex exactly once, and we shall say that $v$ is a normal vertex. To each vertex $v$ we can associate a set $E_{\mathcal{T}}(v)$ containing the end vertices of the unique trail with $v$ as an internal vertex, when such a trail exists in $\mathcal{T}$. When $v$ is eccentric we obviously have $E_{\mathcal{T}}(v)=\emptyset$. It must be clear that we can have $v \in E_{\mathcal{T}}(v)$ since we consider a partition of trails. In Figure 1 we have drawn $K_{4}$ with the trail partition $\mathcal{T}=\{b d a b c, d c, a c\}$. The vertex $c$ is an eccentric vertex while $a, b$ and $d$ are normal vertices.

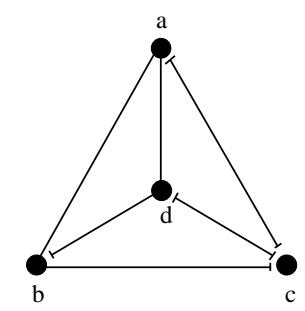

Fig. 1. Normal and eccentric vertices 
Definition 1.1 A partition $\mathcal{T}=\left\{T_{1}, T_{2} \ldots, T_{k}\right\}$ of $E(G)$ into trails is normal when every vertex is normal.

When $\mathcal{T}$ is a normal partition, we can associate to each vertex the unique edge with end $v$ which is the end edge of a trail of $\mathcal{T}$. We shall denote this edge by $e_{\mathcal{T}}(v)$ and it will be convenient to say that $e_{\mathcal{T}}(v)$ is the marked edge associated to $v$. When it will be necessary to illustrate our purpose by a figure the marked edge associated to a vertex will be figurate by a $\vdash$ close to this vertex.

Our purpose, in this paper, is to investigate this new notion of normal partition. In particular we shall see that normal odd partitions can be associated in a natural way to perfect matchings. We shall introduce the notion of compatible normal partitions (to be defined later) leading to a property that could be verified by every bridgeless cubic graph (including the so called snarks) and we shall give some results in that direction.

Definition 1.2 A partition $\mathcal{T}=\left\{T_{1}, T_{2} \ldots, T_{k}\right\}$ of $E(G)$ into trails is odd when every trail in $\mathcal{T}$ is odd.

Definition 1.3 A partition $\mathcal{T}=\left\{T_{1}, T_{2} \ldots, T_{k}\right\}$ of $E(G)$ where each trail is a path will be called a path partition.

Definition 1.4 A partition $\mathcal{P}=\left\{P_{1}, P_{2} \ldots, P_{k}\right\}$ of $V(G)$ into paths is a perfect path partition when every vertex of $G$ is contained in $\mathcal{P}$ (let us note that $k \leq \frac{n}{2}$ ). A perfect matching is thus a perfect path partition where each path has length 1 .

Notations: Following Bondy [1], a walk in a graph $G$ is sequence $W:=$ $v_{0} e_{1} v_{1} \ldots e_{k} v_{k}$, where $v_{0}, v_{1}, \ldots, v_{k}$ are vertices of $G$, and $e_{1}, e_{2} \ldots, e_{k}$ are edges of $G$ and $v_{i-1}$ and $v_{i}$ are the ends of $e_{i}, 1 \leq i \leq k$. The vertices $v_{0}$ and $v_{k}$ are the end vertices and $e_{1}$ and $e_{k}$ are the end edges of this walk, while $v_{1}, \ldots, v_{k-1}$ are the internal vertices and $e_{2}, \ldots, e_{k-1}$ are the internal edges. The length $l(W)$ of $W$ is the number of edges (namely $k$ ). The walk $W$ is odd whenever $k$ is odd and even otherwise.

The walk $W$ is a trail if its edges $e_{1}, e_{2}, \ldots, e_{k}$ are distinct and a path if its vertices $v_{0}, v_{1}, \ldots, v_{k}$ are distinct. If $W:=v_{0} e_{1} v_{1} \ldots e_{k} v_{k}$, is a walk of $G$, $W^{\prime}:=v_{i} e_{i+1} \ldots e_{j} v_{j}(0 \leq i \leq j \leq k)$ is a subwalk of $W$ (subtrails and subpaths are defined analogously).

If $v$ is an internal vertex of a walk $W$ with ends $x$ and $y, W(x, v)$ and $W(v, y)$ are the subwalks of $W$ obtained by cutting $W$ in $v$. Conversely if $W_{1}$ and $W_{2}$ have a common end $v$, the concatenation of these two walks on $v$ gives rise to a new walk (denoted by $W_{1}+W_{2}$ ) with $v$ as an internal vertex. When no 
confusion, is possible, it will be convenient to omit the edges in the description of a walk, that is $W:=v_{0} e_{1} v_{1} \ldots e_{k} v_{k}$ will be shorten in $W:=v_{0} v_{1} \ldots v_{k}$.

When $F \subseteq E(G), V(F)$ is the set of vertices which are incident with some edge of $F$ and $G-F$ is the graph obtained from $G$ by deleting the edges of $F$. A strong matching $C$ in a graph $G$ is a matching $C$ such that there is no edge of $E(G)$ connecting any two edges of $C$, or, equivalently, such that $C$ is the edge-set of the subgraph of $G$ induced on the vertex-set $V(C)$.

\section{$2 \quad$ Elementary properties}

Proposition 2.1 Let $G$ be a cubic graph. Then we can find a normal partition of $E(G)$ within a linear time.

Proof We can easily obtain a partition $\mathcal{T}=\left\{T_{1}, T_{2} \ldots, T_{k}\right\}$ of $E(G)$ into trails via a greedy algorithm. If every vertex is normal then $\mathcal{T}$ is normal and we are done. If $v$ is an eccentric vertex then $v$ is the end vertex of two distinct trails $T_{1}$ and $T_{2}$. Let $T^{\prime}$ be the trail obtained by concatenation of $T_{1}$ and $T_{2}$ on $v$. Then $v$ is an internal vertex of $T^{\prime}$ and $T-\left\{T_{1}, T_{2}\right\}+T^{\prime}$ is a partition of $E(G)$ into trails with one eccentric vertex less (namely $v$ ). This operation can be repeated as long as the current partition into trails has an eccentric vertex and we end with a normal partition in at most $O(n)$ steps.

Proposition 2.2 A partition $\mathcal{T}$ of a cubic graph $G$ is normal if and only if $|\mathcal{T}|=\frac{n}{2}$.

Proof Assume that $\mathcal{T}$ is normal, then every vertex is the end of exactly one trail. Hence $|\mathcal{T}|=\frac{n}{2}$.

Conversely let $\mathcal{T}$ be a partition of the edge set of $G$ into trails. Assume that $|\mathcal{T}|=\frac{n}{2}$ and $T$ is not normal. Then, performing the operation described in Proposition 2.1 on eccentric vertices leads to a normal partition $\mathcal{T}^{\prime}$ such that $\left|\mathcal{T}^{\prime}\right|<\frac{n}{2}$, since the concatenation of two trails on a vertex decreases the number of trails in the partition, a contradiction.

We shall denote by $n_{\mathcal{T}}^{i}$ the number of trails of length $i$ and by $\mu(T)$ the average length of trails in a partition $\mathcal{T}$.

Proposition 2.3 Let $\mathcal{T}$ be a normal partition of a cubic graph $G$ on $n$ vertices. Then 
- $\mu(\mathcal{T})=3$

- $\sum_{i=1}^{i=n+1}(3-i) n_{\mathcal{T}}^{i}=0$

Proof $\mathcal{T}$ being normal, we have $|\mathcal{T}|=\frac{n}{2}$ by Proposition 2.2. Since $|E(G)|=$ $\frac{3 n}{2}$ we have obviously $\mu(\mathcal{T})=3$.

We have

$$
\sum_{i=1}^{i=n+1} i \times n_{\mathcal{T}}^{i}=\frac{3 n}{2}=3 \sum_{i=1}^{i=n+1} n_{\mathcal{T}}^{i}
$$

and hence

$$
\sum_{i=1}^{i=n+1}(3-i) n_{\mathcal{T}}^{i}=0
$$

The length of a normal partition $\mathcal{T}$ (denoted by $l(T)$ ) is the length of the longest trail in $\mathcal{T}$. Let us note that, by Proposition 2.3, every trail of a normal partition $\mathcal{T}$ of $G$ has length 3 when $l(\mathcal{T}) \leq 3$

Proposition 2.4 A cubic graph $G$ on $n$ vertices has an hamiltonian path if and only if $G$ has a normal partition $\mathcal{T}$ such that $l(\mathcal{T})=n+1$

Proof Assume that $P=v_{1} v_{2} \ldots v_{n}$ is an hamiltonian path of $G$. We shall consider that $v_{i}$ is joined to $v_{i+1}$ by the edge $e_{i}$ in $P$. Let $w_{1}\left(w_{n}\right.$ respectively) be a vertex adjacent to $v_{1}$ ( $w_{1}$ respectively) by the edge $e_{1}^{\prime}$ ( $e_{n}^{\prime}$ respectively) not in $E(P)\left(e_{1}^{\prime} \neq e_{n}^{\prime}\right)$. Let $T_{1}$ be the trail $w_{1} e_{1}^{\prime} v_{1} e_{1} v_{2} e_{2} \ldots e_{n-1} v_{n} e_{n}^{\prime} w_{n} . E(G)-T_{1}$ is reduced to a matching of size $\frac{n-2}{2}$ and it can be easily checked that this matching together with $T_{1}$ is a normal partition of $G$ of length $n+1$.

Conversely let $\mathcal{T}$ be a normal partition of $G$ of length $n+1$ and let $T_{1}=$ $w_{1} e_{1} v_{1} e_{1} v_{2} e_{2} \ldots e_{n-1} v_{n} e_{n} w_{n}$ be a trail of maximum length in $T$. Since the only vertices which can appear twice in $T_{1}$ are precisely $w_{1}$ and $w_{n}, P=v_{1} v_{2} \ldots v_{n}$ is an hamiltonian path of $G$.

Theorem 2.5 Let $G$ be a cubic graph having a perfect path partition $\mathcal{P}=$ $\left\{P_{1}, P_{2} \ldots, P_{k}\right\}$. Assume that the ends of $P_{i}$ are $x_{i}$ and $y_{i}$ for every $i=1 \ldots k$. Then $G$ has a normal partition $\mathcal{T}=\left\{T_{1}, T_{2} \ldots, T_{\frac{n}{2}}\right\}$ such that $T_{i}$ is obtained from $P_{i}$ by adding one edge incident to $x_{i}$ and one edge incident to $y_{i}$ for every $i=1 \ldots k$.

Proof The subgraph of $G$ obtained by deleting the edges of each $P_{i}$ is a set of disjoint paths. Let us give an arbitrary orientation to these paths. We get a 
normal partition $\mathcal{T}$ by adding the outgoing edge incident to $x_{i}$ and to $y_{i}$ (for every $i=1 \ldots k$ ), the remaining edges being a set of trails of length 1 in $\mathcal{T}$.

Let $l_{1}, l_{2} \ldots l_{\frac{n}{2}}$ be a set of integers $\left(l_{i} \geq 1\right)$ such that

$$
\sum_{i=1}^{\frac{n}{2}} l_{i}=\frac{3 n}{2} .
$$

Is it possible to find a normal partition $\mathcal{T}=\left\{T_{1}, T_{2} \ldots, T_{\frac{n}{2}}\right\}$ where $l\left(T_{i}\right)=l_{i}$ for every $i=1 \ldots \frac{n}{2}$ ? We do not know the complete answer, however, when $G$ has an hamiltonian cycle we have the following result (an extension of a result of $[2])$ :

Theorem 2.6 Let $G$ be a cubic hamiltonian graph. Let $l_{1}, l_{2} \ldots l_{\frac{n}{2}}$ be a set of integers such that

- $\sum_{i=1}^{\frac{n}{2}} l_{i}=\frac{3 n}{2}$

- $l_{i} \geq 1 \quad l_{i} \neq 2 \forall i=1 \ldots \frac{n}{2}$

Then $G$ has a normal partition $\mathcal{T}=\left\{T_{1}, T_{2} \ldots, T_{\frac{n}{2}}\right\}$ where $l\left(T_{i}\right)=l_{i}$ for every $i=1 \ldots \frac{n}{2}$

Proof Let $\lambda_{i}=l_{i}-2$ and assume that $\lambda_{1} \geq \lambda_{2} \geq \ldots \geq \lambda_{\frac{n}{2}}$. The first $k$ values (for some $k \leq \frac{n}{2}$ ) are greater than 1 , and the remaining values are -1 , since $l_{i} \neq 2$ for all $i=1 \ldots \frac{n}{2}$. We have

$$
\begin{gathered}
\sum_{i=1}^{k} \lambda_{i}=\sum_{i=1}^{k}\left(l_{i}-2\right)=\sum_{i=1}^{k} l_{i}-2 k \\
\sum_{i=1}^{k} l_{i}-2 k=\sum_{i=1}^{k} l_{i}-2 k+\sum_{j=k+1}^{\frac{n}{2}} l_{j}-\left(\frac{n}{2}-k\right)
\end{gathered}
$$

since $\sum_{i=1}^{k} l_{i}+\sum_{j=k+1}^{\frac{n}{2}} l_{j}=\frac{3 n}{2}$ we get that

$$
\sum_{i=1}^{k} \lambda_{i}=n-k
$$

Let $C$ be an hamiltonian cycle of $G$, we can thus arrange a set $\mathcal{P}$ of vertex disjoint paths $P_{i}$ of length $\lambda_{i}(i=1 \ldots k)$ along this cycle. $\mathcal{P}$ is a perfect path partition and, applying Theorem 2.5 we have a normal partition of $G$ as claimed. 
Let $\mathcal{T}$ be a normal partition of a cubic graph $G$ and let $v$ be any vertex of $G$. $E_{\mathcal{T}}(v)$ contains exactly two vertices, namely $x$ and $y$ and one of them, at least, must be distinct from $v$ (we may assume that $v \neq x$ ). Let $T_{1}$ be the trail with ends $x$ and $y$ such that $v$ is an internal vertex of $T_{1}$. Since $\mathcal{T}$ is normal, there is a trail $T_{2}$ ending in $v$ (with the edge $e_{\mathcal{T}}(v)$ ). If $T_{1}^{\prime}$ denotes the trail obtained by concatenation of $T_{1}(x, v)$ and $T_{2}$ on $v$, then $\mathcal{T}-\left\{T_{1}, T_{2}\right\}+T_{1}^{\prime}+T_{1}(v, y)$ is a new normal partition of $G$. We shall say that the above operation is a switch on $v$. When $v \notin E_{\mathcal{T}}(v)$ two such switchings are allowed (see Figure 2), but when $v \in E_{\mathcal{T}}(v)$ only one switching is possible (see Figure 3 ). A switch on a vertex $v$ (leading from a normal partition $\mathcal{T}$ to the normal partition $\mathcal{T}^{\prime}=\mathcal{T} * v$ ) does not change the edge marked associated to $w$ when $w \neq v$. That is $e_{\mathcal{T}}(w)=e_{\mathcal{T}^{\prime}}(w)$. On the other hand the sets $E_{\mathcal{T}^{\prime}}(w)$ may have changed for vertices of $T_{1}$ and $T_{2}$. When $\mathcal{T}$ is a normal odd partition and when $\mathcal{T}^{\prime}=\mathcal{T} * v$ remains an odd partition, the switch on $v$ is said to be an odd switch. It is not difficult to see that, given a normal odd partition, an odd switch is always possible on every vertex.

We shall say that $\mathcal{T}$ and $\mathcal{T}^{\prime}$ are switching equivalent (resp. odd switching equivalent) whenever $\mathcal{T}^{\prime}$ can be obtained from $\mathcal{T}$ by a sequence of switchings (resp. odd switchings). The switching class (resp. odd switching class) of $\mathcal{T}$ is the set of normal partitions which are switching equivalent (resp. odd switching equivalent) to $\mathcal{T}$.
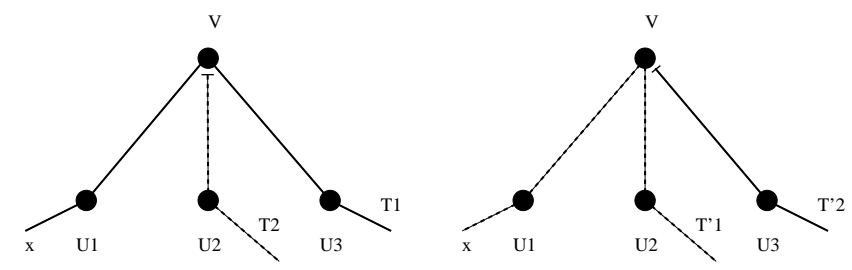

Fig. 2. Switching on $v$ with two distinct trails
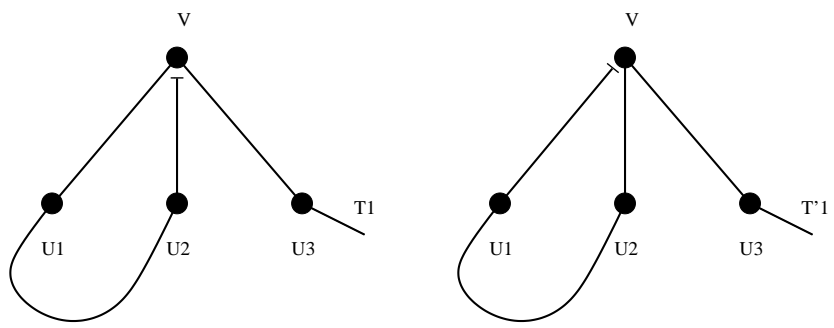

Fig. 3. Switching on $v$ with one trail

Theorem 2.7 Let $G$ be a cubic graph and let $\mathcal{T}$ and $\mathcal{T}^{\prime}$ be any two normal (resp. odd) partitions. Then $\mathcal{T}^{\prime}$ can be obtained from $\mathcal{T}$ by a sequence of (resp. odd) switchings of length at most 2 n.

Proof Let $A_{\mathcal{T} \mathcal{T}^{\prime}}=\left\{v \mid v \in V(G), e_{\mathcal{T}}(v)=e_{\mathcal{T}^{\prime}}(v)\right\}$ and assume that $V(G)-$ $A_{\mathcal{T} \mathcal{T}^{\prime}} \neq \emptyset$ (otherwise we obviously have $\mathcal{T}=\mathcal{T}^{\prime}$ ). We want to pick a vertex 
in $V(G)-A_{\mathcal{T} \mathcal{T}^{\prime}}$ and try to switch the normal partition $\mathcal{T}$ on this vertex (or $\mathcal{T}^{\prime}$ ) in order to increase the size of $A_{\mathcal{T} \mathcal{T}^{\prime}}$ (formally we have changed $\mathcal{T}$ into $\mathcal{T}_{1}$ and $\mathcal{T}^{\prime}$ into $\mathcal{T}_{1}^{\prime}$ and we consider the set $\left.A_{\mathcal{T}_{1} \mathcal{T}_{1}^{\prime}}\right)$. We can suppose that $\mathcal{T}$ and $\mathcal{T}^{\prime}$ are not switching equivalent and, moreover, among the switching equivalent normal partitions of $\mathcal{T}$ and those of $\mathcal{T}^{\prime}, A_{\mathcal{T} \mathcal{T}^{\prime}}$ has maximum cardinality.

Let $v \notin A_{\mathcal{T} \mathcal{T}^{\prime}}$ and let $e_{1}, e_{2}$ and $e_{3}$ be the edges adjacent to $v$. Assume that $e_{\mathcal{T}}(v)=e_{1}$ and $e_{\mathcal{T}^{\prime}}(v)=e_{2}$. Recall that in both partitions a switch (resp. odd switch) is always possible on $v$.

Consider first a possible switch (resp. odd switch) on $v$ in $\mathcal{T}$, we get hence a new normal partition $\mathcal{T} * v$. If $e_{\mathcal{T} * v}=e_{2}$ then $A_{\mathcal{T} * v, \mathcal{T}^{\prime}}=A_{\mathcal{T}, \mathcal{T}^{\prime}} \cup\{v\}$, a contradiction. If by switching (resp. odd switching) $\mathcal{T}^{\prime}$ on $v$ we have $e_{\mathcal{T}^{\prime} * v}=e_{1}$ then $A_{\mathcal{T}, \mathcal{T}^{\prime} * v}=A_{\mathcal{T}, \mathcal{T}^{\prime}} \cup\{v\}$, a contradiction. Finally, if $e_{\mathcal{T} * v} \neq e_{2}$ and $e_{\mathcal{T}^{\prime} * v} \neq e_{1}$ that means that $e_{\mathcal{T}_{* v}}=e_{3}$ and $e_{\mathcal{T}^{\prime * v}}=e_{3}$, thus $A_{\mathcal{T}^{*}, \mathcal{T}^{\prime} * v}=A_{\mathcal{T}, \mathcal{T}^{\prime}} \cup\{v\}$, a contradiction.

Hence any two normal partitions are switching equivalent (resp. odd switching equivalent). In order to increase the size of $A_{\mathcal{T} \mathcal{T}^{\prime}}$, we have seen that we eventually are obliged to proceed to two switchings on the same vertex (one with $\mathcal{T}$ and one with $\mathcal{T}^{\prime}$ ). It is clear that we need at most $2 n$ such switchings on the road leading to $\mathcal{T}^{\prime}$ from $\mathcal{T}$.

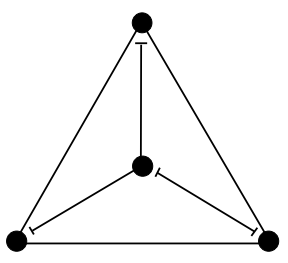

Fig. 4. No normal partitions associated to the $\vdash$

In Figure 4, we can see that it is not possible to find a normal partition of $K_{4}$ for which the set of marked edges is given by those having a $\vdash$ at one end. Since the set of edges with no end marked contains a cycle the following question is thus natural. Given a set of edges $F=\left\{e_{v} \mid v \in V(G)\right\}$, where each vertex of $V(G)$ appears exactly once as the end of an edge of $F$, under which condition can we say that this set of edges is the set of marked edges associated to a normal partition?

Theorem 2.8 Let $F$ be a set of edges of $G$, where each vertex of $V(G)$ appears exactly once as the end of an edge of $F$. Then there exists a normal partition $\mathcal{T}$ such that $F$ is the set of marked edges associated to $\mathcal{T}$ if and only if $F$ is a transversal of the cycles of $G$.

Proof Let $\mathcal{T}$ be a normal partition, the set of marked edges $\left\{e_{T}(v) \mid v \in V(G)\right\}$ is obviously a transversal of the cycles of $G$, since $\mathcal{T}$ is partitioned into trails. 
Conversely, assume that $F=\left\{e_{v} \mid v \in V(G)\right\}$ is a transversal of the cycles of $G$.

Then the spanning subgraph $G-F$ is a set of paths $\left\{P_{1}, P_{2}, \ldots, P_{k}\right\}$ (some of them being eventually reduced to a vertex). Let $u_{i}$ and $v_{i}$ be the end vertices of $P_{i}(1 \leq i \leq k)$ (when $P_{i}$ is reduced to a single vertex, we have $\left.u_{i}=v_{i}\right)$. We add to each path $P_{i}$ the edges of $F$ which are incident to $u_{i}$ and $v_{i}$ and distinct from $e_{u_{i}}$ and $e_{v_{i}}$. We get a set of trails $\mathcal{T}=\left\{T_{1}, T_{2}, \ldots, T_{k}\right\}$ which partition the edge set. We claim that $\mathcal{T}$ is a normal partition. Indeed, let $v$ be any vertex of $G$. The vertex $v$ is contained in some path $P_{i}$ of $G-F$ and $T_{i}$ must contain the two edges incident to $v$ and distinct from the unique edge associated to $v$ in $F$. Hence $v$ must be an internal vertex of $T_{i}$ which implies that $v$ is normal.

\section{On compatible normal partitions}

Definition 3.1 Two partitions $\mathcal{T}=\left\{T_{1}, T_{2} \ldots, T_{k}\right\}$ and $\mathcal{T}^{\prime}=\left\{T_{1}^{\prime}, T_{2}^{\prime} \ldots, T_{k}^{\prime}\right\}$ of $E(G)$ into trails are compatible when $e_{T}(v) \neq e_{T^{\prime}}(v)$ for every vertex $v \in V(G)$.

Theorem 3.2 Let $G$ be a cubic graph. Then the three following statements are equivalent.

i) G has a perfect matching

ii) $G$ has an odd normal partition

iii) $G$ has two compatible normal partitions of length 3

\section{Proof}

Let $M$ be a perfect matching in $G$. Then $G-M$ is a 2 -factor of $G$. Let us give any orientation to the cycles of this 2 -factor and for each vertex $v$ let us denote the outgoing edge $o(v)$. For each edge $e=u v \in M$, let $P_{u v}$ be the trail of length 3 obtained by concatenation of $o(u), u v$ and $o(v)$. Then $\mathcal{T}=\left\{P_{u v} \mid u v \in M\right\}$ is a normal odd partition (of length 3 ) of $G$. We obtain a second normal partition $\mathcal{T}^{\prime}$ of length 3 , compatible with $\mathcal{T}$, when we choose the other orientation on each cycle. Hence (i) implies (ii) and (iii).

Let $\mathcal{T}=\left\{T_{1}, T_{2} \ldots, T_{\frac{n}{2}}\right\}$ be a normal odd partition of $G$. For each trail $T_{i} \in T$ let us say that an edge $e$ of $T_{i}$ is odd whenever the subtrails of $T_{i}$ obtained by deleting $e$ have odd lengths (an even edge being defined in the similar way). Any vertex $v \in V(G)$ is internal in exactly one trail of $\mathcal{T}$. The edges of this trail being alternatively odd and even, $v$ is incident to exactly one odd edge. Hence the odd edges so defined induce a perfect matching of $G$ and (ii) implies (i). 
Since (iii) implies obviously (ii), the proof is complete.

Definition 3.3 A Perfect Path Double Cover (PPDC for short) is a collection $\mathcal{P}$ of paths such that each edge of $G$ belongs to exactly two members of $\mathcal{P}$ and each vertex occurs exactly twice as an end path of $\mathcal{P}$.

This notion has been introduced by Bondy (see [1]) who conjectured that every simple graph admits a $P P D C$. This conjecture was proved by $\mathrm{Li}[9]$. When dealing with two compatible normal path partitions $\mathcal{P}$ and $\mathcal{P}^{\prime}$ in a cubic graph, we have a particular $P P D C$. Indeed every edge belongs to exactly one path of $\mathcal{P}$ and one path of $\mathcal{P}^{\prime}$ and every vertex occurs exactly once as an end vertex of a path in $\mathcal{P}$ and a path in $\mathcal{P}^{\prime}$. The qualifying adjective compatible says that the two end edges are distinct for each vertex.

As a refinement of the notion of $P P D C$ we can define a $C P P D C$ for a simple graph:

Definition 3.4 A Compatible Perfect Path Double Cover (CPPDC for short) is a collection $\mathcal{P}$ of paths such that each edge of $G$ belongs to exactly two members of $\mathcal{P}$ and each vertex occurs exactly twice as an end path of $\mathcal{P}$ and these two ends are distinct.

A natural question is thus to know which graphs admits a $C P P D C$. If we restrict ourself to connected graphs, we immediately can see that as soon as a graph has a pendent edge, a $C P P D C$ does not exist. We need thus to consider graphs with a certain connectivity condition. As an easy result we see that a minimal 2-edge connected graph has $C P P D C$.

Proposition 3.5 Let $G$ ba a minimal 2-edge connected simple graph. Then $G$ admits a $C P P D C$.

Proof By induction on the number of vertices. The assertion can be verified on the complete graph with three vertices, so assume that $G$ has at least four vertices. It is well known (see Halin [6]) that $G$ contains a vertex $v$ whose degree is 2. Let $v_{1}$ and $v_{2}$ be the two neighbors of $v$.

case 1: $v_{1} v_{2} \in E(G)$.

Let $G^{\prime}$ be the graph obtained from $G$ by deleting $v$ and the edge $v_{1} v_{2}$. Since $G$ is minimal 2-edge connected, $G^{\prime}$ has 2 connected component $C_{i}(i=1,2)$, with $v_{i} \in C_{i}$. We can see that these subgraphs are minimal 2-edge connected. We can thus find a $C P P D C \mathcal{T}_{i}(i=1,2)$ for each of them. Let $Q_{i}, R_{i} \in \mathcal{T}_{i}$ $(i=1,2)$ be the two paths with end vertices $v_{i}$. Let $T_{1}=Q_{1}+v_{1} v_{2} v$ and 
$T_{2}=Q_{2}+v_{2} v_{1} v$. Then $\mathcal{T}=\mathcal{T}_{1}-Q_{1}+\mathcal{T}_{2}-Q_{2}+\left\{T_{1}, T_{2}\right\}+v_{1} v v_{2}$ is a $C P P D C$ of $G$.

case 2: $v_{1} v_{2} \notin E(G)$ and $G-v$ is not minimal 2-edge connected.

Let $G^{\prime}$ be the graph obtained from $G$ by adding the edge $v_{1} v_{2}$ and deleting the vertex $v$.

Assume that $G^{\prime}$ is still a minimal $2-$ edge connected. Then let $\mathcal{T}^{\prime}$ be a $C P P D C$ of $G^{\prime}$ and let $T_{1}^{\prime}, T_{2}^{\prime} \in \mathcal{T}^{\prime}$ be the two paths using the edge $v_{1} v_{2}$. We can transform this $C P P D C$ of $G^{\prime}$ in a $C P P D C$ of $G$ when we consider $\mathcal{T}=$ $\mathcal{T}^{\prime}-\left\{T_{1}^{\prime}, T_{2}^{\prime}\right\}+\left\{T_{1}^{1}, T_{1}^{2}, T_{2}\right\}$ where $T_{2}$ is obtained from $T_{2}^{\prime}$ by inserting $v$ between $v_{1}$ and $v_{2}$ and $T_{1}^{1}, T_{1}^{2}$ are obtained from $T_{1}^{\prime}$ by deleting the edge $v_{1} v_{2}$ and adding the edge $v_{1} v$ to the subpath of $T_{1}^{\prime}$ containing $v_{1}$ (respectively, the edge $v_{2} v$ to the subpath of $T_{2}^{\prime}$ containing $v_{2}$ ).

When $G^{\prime}$ is not a minimal 2-edge connected graph, there is an edge of $G^{\prime}$ whose deletion preserves the 2 -edge connectivity. In fact, we can check that the only edge with that property must be the edge $v_{1} v_{2}$ (otherwise $G$ itself is not minimal 2-edge connected). A contradiction since we have supposed that $G-v$ is not minimal 2-edge connected.

case 3: $v_{1} v_{2} \notin E(G)$ and $G-v$ is minimal 2-edge connected.

Let $G^{\prime}=G-v$ and let $\mathcal{T}^{\prime}$ be a $C P P D C$ of $G^{\prime}$. Let $Q_{i}, R_{i} \in \mathcal{T}^{\prime}(i=1,2)$ be the two paths with end vertices $v_{i}$. We can consider that $Q_{1}$ and $Q_{2}$ are two distinct paths of $\mathcal{T}^{\prime}$. Then, let $\mathcal{T}=\mathcal{T}^{\prime}-\left\{Q_{1}, Q_{2}\right\}+\left\{T_{1}, T_{2}\right\}+v_{1} v v_{2}$ where $T_{i}$ is obtained by concatenation of $Q_{i}$ and $v_{i} v(i=1,2)$. We can check that $\mathcal{T}$ is a $C P P D C$ of $G$.

We propose as an open Problem

Problem 3.6 Every 2-edge connected simple graph admits a CPPDC.

Remark 3.7 Assume that a connected graph $G$ admits $C P P D C$. In doubling every edge $e$ in $e^{\prime}$ and $e^{\prime \prime}$ (let $G_{2}$ the graph so obtained), this CPPDC leads to an euler tour of $G_{2}$. This euler tour is compatible (in the sense given by Kotzig [8]) with the set of transitions defined by $e^{\prime}$ and $e^{\prime \prime}$ in each vertex. 


\section{On three compatible normal partitions}

We shall say that $G$ has three compatible normal partitions $\mathcal{T}, \mathcal{T}^{\prime}$ and $\mathcal{T}^{\prime \prime}$ whenever these partitions are pairwise compatible.

NB: As usual $N(v)$ denotes the set of vertices adjacent to $v$.

Theorem 4.1 A cubic graph $G$ has three compatible normal partitions if and only if $G$ has no loop.

Proof Let $G$ be a cubic graph with three compatible normal partitions $\mathcal{T}$, $\mathcal{T}^{\prime}$ and $\mathcal{T}^{\prime \prime}$. Assume that $G$ contains a loop $v v$, let $w \neq v$ be the vertex adjacent to $v$. Then one of these normal partitions, say $\mathcal{T}$, would be such that $e_{\mathcal{T}}(v)=v w$. In that case $v v$ would be the trail containing $v$ as an internal vertex, impossible.

Conversely, assume that $G$ has no loop and $G$ can not be provided with three compatible normal partitions. We can suppose that $G$ has been chosen with the minimum number of vertices for that property. Figure 5 shows that $G$ has certainly at least 4 vertices.

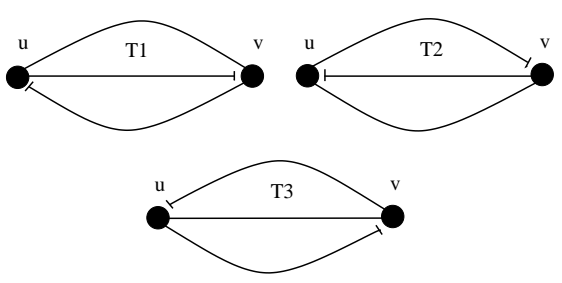

Fig. 5. Cubic graph on 2 vertices with three compatible normal partitions

CLAIM 1 If $u$ and $v$ are joined by two edges $e_{1}$ and $e_{2}$, then there is a third vertex $w$ adjacent to $u$ and $v$.

Proof Assume that $u$ is adjacent to $u^{\prime}$ and $v$ to $v^{\prime}$ with $u^{\prime} \neq u$ and $v^{\prime} \neq v$. Let $G^{\prime}$ be the cubic graph obtained from $G$ by deleting $u$ and $v$ and joining $u^{\prime}$ and $v^{\prime}$ by a new edge. $G^{\prime}$ is obviously a cubic graph with no loop and $|V(G)|<\left|V\left(G^{\prime}\right)\right|$. We can thus find three compatible normal partitions $\mathcal{T}, \mathcal{T}^{\prime}$ and $\mathcal{T}^{\prime \prime}$ in $G^{\prime}$.

The edge $u^{\prime} v^{\prime}$ of $G^{\prime}$ is contained into $T \in \mathcal{T}, T^{\prime} \in \mathcal{T}^{\prime}$ and $T^{\prime \prime} \in \mathcal{T}^{\prime \prime}$. For convenience, $T_{1}$ and $T_{2}$ will be the subtrails of $T$ we have obtained by deleting $u^{\prime} v^{\prime}$, with $u^{\prime}$ an end of $T_{1}$ and $v^{\prime}$ an end of $T_{2}$. Following the same trick we get $T_{1}^{\prime}$ and $T_{2}^{\prime}, T_{1}^{\prime \prime}$ and $T_{2}^{\prime \prime}$ when considering $T^{\prime}$ and $T^{\prime \prime}$. It can be noticed that some of these subtrails may have length 0 , which means that, following the cases, $u v$ is the marked edge associated to $u$ or (and) $v$ in $\mathcal{T}, \mathcal{T}^{\prime}$ or $\mathcal{T}^{\prime \prime}$. 
Let $P_{1}=T_{1}+u^{\prime} u, P_{2}=T_{2}+v^{\prime} v e_{1} u e_{2} v$ and $\mathcal{Q}=\mathcal{T}-P+\left\{P_{1}, P_{2}\right\}$. We can easily check that $\mathcal{Q}$ is a normal partition of $G$ where $e_{\mathcal{Q}}(x)=e_{\mathcal{T}}(x) \forall x \neq u, v$ and $e_{\mathcal{Q}}(u)=u u^{\prime}, e_{\mathcal{Q}}(v)=e_{2}$.

In the same way, let $P_{1}^{\prime}=T_{1}^{\prime}+u^{\prime} u e_{2} v e_{1} u, P_{2}^{\prime}=T_{2}^{\prime}+v^{\prime} v$ and $\mathcal{Q}^{\prime}=\mathcal{T}^{\prime}-P^{\prime}+$ $\left\{P_{1}^{\prime}, P_{2}^{\prime}\right\}$. Then $e_{\mathcal{Q}^{\prime}}(x)=e_{\mathcal{T}^{\prime}}(x) \forall x \neq u, v$ and $e_{\mathcal{Q}^{\prime}}(u)=e_{1}^{\prime}, e_{\mathcal{Q}^{\prime}}(v)=v v^{\prime}$. Hence $\mathcal{Q}^{\prime}$ is a normal partition compatible with $\mathcal{Q}$.

Finally, let $P_{1}^{\prime \prime}=T_{1}^{\prime \prime}+u^{\prime} u e_{1} v, P_{2}^{\prime \prime}=T_{2}^{\prime}+v^{\prime} v e_{2} u$ and $\mathcal{Q}^{\prime \prime}=\mathcal{T}^{\prime \prime}-P^{\prime \prime}+\left\{P_{1}^{\prime \prime}, P_{2}^{\prime \prime}\right\}$. Then $e_{\mathcal{Q}^{\prime \prime}}(x)=e_{\mathcal{T}^{\prime \prime}}(x) \forall x \neq u, v$ and $e_{\mathcal{Q}^{\prime \prime}}(u)=e_{2}, e_{\mathcal{Q}^{\prime \prime}}(v)=e_{1}$. Hence $\mathcal{Q}, \mathcal{Q}^{\prime}$ and $\mathcal{Q}^{\prime \prime}$ are three compatible normal partitions of $G$, a contradiction.

Claim 2 if $u v \in E(G)$ then $|N(u)|=2$ or $|N(v)|=2$

Proof Assume that $|N(u)|=3$ and $|N(v)|=3$ and let $u^{\prime}$ and $u^{\prime \prime}$ the two neighbors of $u$ and $v^{\prime}$ and $v^{\prime \prime}$ those of $v$. Let $G^{\prime}$ be the graph obtained from $G$ by deleting $u$ and $v$ and joining $u^{\prime}$ and $u^{\prime \prime}$ by a new edge as well as joining $v^{\prime}$ and $v^{\prime \prime} . G^{\prime}$ is obviously a cubic graph with no loop and $|V(G)|<\left|V\left(G^{\prime}\right)\right|$. We can thus find three compatible normal partitions $\mathcal{T}, \mathcal{T}^{\prime}$ and $\mathcal{T}^{\prime \prime}$ in $G^{\prime}$.

The edge $u^{\prime} u^{\prime \prime}$ of $G^{\prime}$ is contained into $T \in \mathcal{T}, T^{\prime} \in \mathcal{T}^{\prime}$ and $T^{\prime \prime} \in \mathcal{T}^{\prime \prime}$ and we denote, as in the previous claim by $T_{1}, T_{2}, T_{1}^{\prime}, T_{2}^{\prime}, T_{1}^{\prime \prime}$ and $T_{2}^{\prime \prime}$ the subtrails of $T, T^{\prime}$ and $T^{\prime \prime}$ obtained by deleting $u^{\prime} u^{\prime \prime}$ (with $u^{\prime}$ an end of trails with subscript 1 and $u^{\prime \prime}$ an end of trails with subscript 2). If $R \in \mathcal{T}, R^{\prime} \in \mathcal{T}^{\prime}$ and $R^{\prime \prime} \in \mathcal{T}^{\prime \prime}$ are the trails using $v^{\prime} v^{\prime \prime}$, we can define also $R_{1}, R_{2}, R_{1}^{\prime}, R_{2}^{\prime}, R_{1}^{\prime \prime}$ and $R_{2}^{\prime \prime}$.

We are going to construct three normal partition $\mathcal{Q}, \mathcal{Q}^{\prime}$ and $\mathcal{Q}^{\prime \prime}$ of $G$ by transforming locally $\mathcal{T}, \mathcal{T}^{\prime}$ and $\mathcal{T}^{\prime \prime}$ in such a way that $e_{\mathcal{Q}}(x)=e_{\mathcal{T}}(x) e_{\mathcal{Q}^{\prime}}(x)=$ $e_{\mathcal{T}^{\prime}}(x)$ and $e_{\mathcal{Q}^{\prime \prime}}(x)=e_{\mathcal{T}^{\prime \prime}}(x) \forall x \neq u, v$. The verification of this point, left to the reader, is immediate.

Let $P_{1}^{\prime \prime}=T_{1}^{\prime \prime}+u^{\prime} u u^{\prime \prime}+T_{2}^{\prime \prime}, P_{2}^{\prime \prime}=R_{1}^{\prime \prime}+v^{\prime} v v^{\prime \prime}+R_{2}^{\prime \prime}$ and $P_{3}^{\prime \prime}=u v \cdot \mathcal{Q}^{\prime \prime}$ is then $\mathcal{T}^{\prime \prime}-\left\{P^{\prime \prime}, R^{\prime \prime}\right\}+\left\{P_{1}^{\prime \prime}, P_{2}^{\prime \prime}, P_{3}^{\prime \prime}\right\}$. We can remark that we have subdivided $P^{\prime \prime}$ and $R^{\prime \prime}$ an we have added a trail of length one $(u v)$. We have hence, $e_{\mathcal{Q}^{\prime \prime}}(u)=u v$ and $e_{\mathcal{Q}^{\prime \prime}}(v)=u v$.

It must be clear that we may have $T=R$ in $\mathcal{T}$, which means that $u^{\prime} u^{\prime \prime}$ and $v^{\prime} v^{\prime \prime}$ are contained in the same trail of $\mathcal{T}$. But we certainly have either $T_{1} \neq R_{1}$ or $T_{1} \neq R_{2}$ since $R_{1}$ and $R_{2}$ are two disjoint trails. Let us consider the following partitions of the edge set of $G$ :

$$
\begin{aligned}
& \mathcal{Q}_{1}=\mathcal{T}-\left\{T_{1}, T_{2}\right\}+\left\{T_{1}+u^{\prime} u v v^{\prime}+R_{1}, T_{2}+u^{\prime \prime} u, R_{2}+v^{\prime \prime} v\right\} \\
& \mathcal{Q}_{2}=\mathcal{T}-\left\{T_{1}, T_{2}\right\}+\left\{T_{1}+u^{\prime} u v v^{\prime \prime}+R_{2}, T_{2}+u^{\prime \prime} u, R_{2}+v^{\prime} v\right\}
\end{aligned}
$$




$$
\begin{aligned}
& \mathcal{Q}_{3}=\mathcal{T}-\left\{T_{1}, T_{2}\right\}+\left\{T_{1}+u^{\prime} u, R_{1}+v^{\prime} v u u^{\prime \prime}+T_{2}, R_{2}+v^{\prime \prime} v\right\} \\
& \mathcal{Q}_{4}=\mathcal{T}-\left\{T_{1}, T_{2}\right\}+\left\{T_{1}+u^{\prime} u, R_{1}+v^{\prime} v, T_{2}+u^{\prime \prime} u v v^{\prime \prime}+R_{2}\right\}
\end{aligned}
$$

$\mathcal{Q}_{1}$ is a normal partition of $G$ as soon as $T_{1} \neq R_{1}$ and we can check, in that case, that $\mathcal{Q}_{2}, \mathcal{Q}_{3}$ and $\mathcal{Q}_{4}$ are normal partitions of $G$. In the same way, $\mathcal{Q}_{2}$ is a normal partition of $G$ as soon as $T_{1} \neq R_{2}$ and we can check, in that case, that $\mathcal{Q}_{1}, \mathcal{Q}_{3}$ and $\mathcal{Q}_{4}$ are normal partitions of $G . \mathcal{Q}_{3}$ is a normal partition of $G$ as soon as $T_{2} \neq R_{1}$ and, in that case, $\mathcal{Q}_{1}, \mathcal{Q}_{2}$ and $\mathcal{Q}_{4}$ are normal partitions of $G$. $\mathcal{Q}_{4}$ is a normal partition of $G$ as soon as $T_{2} \neq R_{2}$ and, in that case, $\mathcal{Q}_{1}$, $\mathcal{Q}_{2}$ and $\mathcal{Q}_{3}$ are normal partitions of $G$.

We can define analogously $\mathcal{Q}_{1}^{\prime}, \mathcal{Q}_{2}^{\prime}, \mathcal{Q}_{3}^{\prime}$ and $\mathcal{Q}_{4}^{\prime}$ when considering $\mathcal{T}^{\prime}$.

We can check moreover that these normal partitions (when they are well defined) $\mathcal{Q}_{1}, \mathcal{Q}_{2}, \mathcal{Q}_{3}, \mathcal{Q}_{4}, \mathcal{Q}_{1}^{\prime}, \mathcal{Q}_{2}^{\prime}, \mathcal{Q}_{3}^{\prime}$ and $\mathcal{Q}_{4}^{\prime}$ are compatible with $\mathcal{Q}^{\prime \prime}$ since

$$
\begin{aligned}
& e_{\mathcal{Q}_{i}}(u)=u u^{\prime} \text { or } e_{\mathcal{Q}_{i}}(u)=u u^{\prime \prime} i=1,2,3,4 \\
& e_{\mathcal{Q}_{i}}(v)=v v^{\prime} \text { or } e_{\mathcal{Q}_{i}}(v)=v v^{\prime \prime} i=1,2,3,4 \\
& e_{\mathcal{Q}_{i}^{\prime}}(u)=u u^{\prime} \text { or } e_{\mathcal{Q}_{i}^{\prime}}(u)=u u^{\prime \prime} i=1,2,3,4 \\
& e_{\mathcal{Q}_{i}^{\prime}}(v)=v v^{\prime} \text { or } e_{\mathcal{Q}_{i}^{\prime}}(v)=v v^{\prime \prime} i=1,2,3,4
\end{aligned}
$$

We can verify that in each case to be considered with $\mathcal{T}\left(T_{1}=R_{1}\right.$ and $T_{2} \neq R_{2}, T_{2}=R_{2}$ and $T_{1} \neq R_{1}, T_{1}=R_{2}$ and $T_{2} \neq R_{1}, T_{2}=R_{1}$ and $T_{1} \neq R_{2}, T_{1}, T_{2}, R_{1}, R_{2}$ all distinct) together with the similar cases for $\mathcal{T}^{\prime}$ we can choose a normal partition $\mathcal{Q}$ in $\left\{\mathcal{Q}_{1}, \mathcal{Q}_{2}, \mathcal{Q}_{3}, \mathcal{Q}_{4}\right\}$ and a normal partition $\mathcal{Q}^{\prime}$ in $\left\{\mathcal{Q}_{1}^{\prime}, \mathcal{Q}_{2}^{\prime}, \mathcal{Q}_{3}^{\prime}, \mathcal{Q}_{4}^{\prime}\right\}$ which are compatible and hence three normal partitions compatible $\mathcal{Q}, \mathcal{Q}^{\prime}$ and $\mathcal{Q}^{\prime \prime}$ for $G$, a contradiction.

Assume that $u$ and $v$ are joined by two edges in $G$, then, from Claim 1, there is unique new vertex $w$ joined to $u$ and $v$. This vertex is adjacent to $x \neq u, v$ which have itself a neighbor $z \neq u, v$. Since $|N(w)|=2$, by Claim 2, $N(x)=\{w, z\}$. The vertices $x$ and $z$ being joined by two edges, $x$ and $z$ must have a common neighbor by Claim 1, impossible. Hence $G$ does not exist and the proof is complete.

Proposition 4.2 Let $G$ be a cubic graph having three compatible normal partitions then every edge $e \in E(G)$ verifies exactly one of the followings

- $e$ is an internal edge in exactly one partition

- $e$ is an internal edge in exactly two partitions

Moreover, in the second case, the edge e itself is a trail of the third partition. 
Proof Let $e=x y$ be any edge of $G$ and let $\mathcal{T}, \mathcal{T}^{\prime}$ and $\mathcal{T}^{\prime \prime}$ be three compatible normal partitions. If $e$ is not an internal edge in $\mathcal{T}, \mathcal{T}^{\prime}$ nor $\mathcal{T}^{\prime \prime}$ then $e$ is an end edge for a trail of $\mathcal{T}, \mathcal{T}^{\prime}$ and $\mathcal{T}^{\prime \prime}$. In $x$ or $y$ we should have two partitions (say $\mathcal{T}$ and $\mathcal{T}^{\prime}$ ) for which $e_{\mathcal{T}}(x)=e_{\mathcal{T}^{\prime}}(x)\left(e_{\mathcal{T}}(y)=e_{\mathcal{T}^{\prime}}(y)\right.$ respectively), a contradiction. So let us suppose that $e$ is an internal edge in $\mathcal{T}, \mathcal{T}^{\prime}$ and $\mathcal{T}^{\prime \prime}$. Let $a$ and $b$ the two other neighbors of $x$. We should have then

- $e_{\mathcal{T}}(x)=x a$ or $x b$

- $e_{\mathcal{T}^{\prime}}(x)=x a$ or $x b$

- $e_{\mathcal{T}^{\prime \prime}}(x)=x a$ or $x b$

which is impossible since the three partitions are compatible. Assume now that $e$ is an internal edge of a trail in $\mathcal{T}$ and in $\mathcal{T}^{\prime}$ and let $a$ and $b$ the two other neighbors of $x$. Up to the names of vertices we have

- $e_{\mathcal{T}}(x)=x a$

- $e_{\mathcal{T}^{\prime}}(x)=x b$

From the third partition $\mathcal{T}^{\prime \prime}$, we must have $e_{\mathcal{T}^{\prime \prime}}(x)=x y$. In the same way we should obtain $e_{\mathcal{T}^{\prime \prime}}(y)=y x$. Hence the trail containing $e=x y$ is reduced to $e$, as claimed.

It can be noticed that whenever a cubic graph can be provided with three compatible normal partitions at least one edge is the internal edge in exactly one partition.

Proposition 4.3 Let $G$ be a cubic graph having three compatible normal partitions. Then at least one edge $e \in E(G)$ is the internal edge in exactly one partition.

Proof Let $\mathcal{T}, \mathcal{T}^{\prime}$ and $\mathcal{T}^{\prime \prime}$ be three compatible normal partitions of $G$. The set of trails of length 1 in $\mathcal{T}$ is a matching of $G$ which means that $\mathcal{T}$ has at most $\frac{n}{2}$ such trails. If each edge of $G$ is the internal edge in exactly two partitions we must have

$$
|E(G)|=n_{\mathcal{T}}^{1}+n_{\mathcal{T}^{\prime}}^{1}+n_{\mathcal{T}^{\prime \prime}}^{1} \leq 3 \frac{n}{2}=|E(G)|
$$

Hence the set of edges which are trails of length 1 in $\mathcal{T}$ is a perfect matching $M$ of $G$. In that case, the set of marked edges associated to $\mathcal{T}$ is precisely this set $M$, which is not transversal of the cycles of $G$, a contradiction with Theorem 2.8.

Theorem 4.4 Let $G$ be a simple 3-edge colourable cubic graph then $G$ has three compatible normal partitions $\mathcal{T}, \mathcal{T}^{\prime}$ and $\mathcal{T}^{\prime \prime}$ such that 
- $\mathcal{T}$ is odd

- $\mathcal{T}^{\prime}$ has length 3

- $\mathcal{T}^{\prime \prime}$ has length 4

Proof In [4], it is proved that, given a 3-edge colouring of $G$ with $\alpha, \beta$ and $\gamma$ then there exists a strong matching intersecting every cycle belonging to the 2factor induced by the two colours $(\alpha$ and $\beta)$. Assume that $\mathcal{C}=\left\{C_{1}, C_{2}, \ldots C_{k}\right\}$ is such a 2 -factor $\left(G-\mathcal{C}\right.$ is a perfect matching) and let $F=\left\{u_{i} v_{i} \in C_{i} \mid 1 \leq\right.$ $i \leq k)$ (minimal for the inclusion) be a strong matching intersecting each cycle of this 2 -factor.

For each $u_{i} v_{i} \in F, x_{i}$ is the vertex in the neighborhood of $u_{i}$ which is not one of its neighbor (predecessor or successor) on $C_{i}$ while $y_{i}$ is defined similarly for $v_{i}$ (note that $x_{i}$ and $y_{i}$ may be vertices of $C_{i}$ or not). Let $T_{i}$ be the trail obtained from $C_{i}$ by adding the edge $u_{i} x_{i}$ and considering that this trail ends with $v_{i} u_{i}$ (Note that $u_{i}$ is an internal vertex of $T_{i}$ ).

Let $\mathcal{T}$ be the trail partition containing every trail $T_{i}(1 \leq i \leq k)$ and all the edges of the perfect matching $G-\mathcal{C}$ which are not in some $T_{i}$. We can check that $\mathcal{T}$ is a normal odd partition for which the following holds

- $e_{\mathcal{T}}\left(u_{i}\right)=u_{i} v_{i}$

- $e_{\mathcal{T}}\left(x_{i}\right)=x_{i} u_{i}$

- $e_{\mathcal{T}}(v)$ is the edge of $G-\mathcal{C}$ for each vertex $v \neq u_{i}, v_{i}$

We construct now the trail partition $\mathcal{T}^{\prime}$. Let us give the orientation to each cycle of $\mathcal{C}$. This orientation is such that the successor of $u_{i}$ is $v_{i}$. For each vertex $v, o(v)$ denotes the successor of $v$ in that orientation and $p(v)$ its predecessor. As in the proof of Theorem 3.2 we get hence a normal partition $\mathcal{T}^{\prime}$ where each trail is a path of length 3. Moreover $e_{\mathcal{T}^{\prime}}(v)=v p(v)$ for every vertex $v$.

Before constructing $\mathcal{T}^{\prime \prime}$, we construct $\mathcal{T}^{\prime \prime \prime}$ by using the reverse orientation on each cycle of $\mathcal{C}$. This normal partition of length 3 is such that $e_{\mathcal{T}^{\prime \prime \prime}}(v)=v o(v)$.

For each vertex $v \neq u_{i} 1 \leq i \leq k$ we have $e_{\mathcal{T}}(v) \neq e_{\mathcal{T}^{\prime}}(v) \neq e_{\mathcal{T}^{\prime \prime \prime}}(v)$.

For $v=u_{i} 1 \leq i \leq k$, we have $e_{\mathcal{T}}\left(u_{i}\right)=u_{i} v_{i}$, $e_{\mathcal{T}^{\prime}}\left(u_{i}\right)=u_{i} p\left(u_{i}\right)$ ) (where $\left.p\left(u_{i}\right) \neq v_{i}\right)$ and $e_{\mathcal{T}^{\prime \prime \prime}}\left(u_{i}\right)=u_{i} v_{i}$. Since $e_{\mathcal{T}}\left(u_{i}\right)=e_{\mathcal{T}^{\prime \prime \prime}}\left(u_{i}\right), \mathcal{T}$ and $\mathcal{T}^{\prime \prime \prime}$ are not compatible.

Our goal now is to proceed to switchings on $\mathcal{T}^{\prime \prime \prime}$ in each vertex $u_{i}$ in order to get $\mathcal{T}^{\prime \prime}$ where these incompatibilities are dropped. For this purpose, we extend every path of length 3 of $\mathcal{T}^{\prime \prime \prime}$ ending with $v_{i} u_{i}$ with the edge $u_{i} p\left(u_{i}\right)$. We get hence of path of length 4 and, since $F$ is a strong matching, we are sure that we cannot extend this path in the other direction. The path of $\mathcal{T}^{\prime \prime \prime}$ ending with $u_{i} p\left(u_{i}\right)$ is shorten by deleting the edge $u_{i} p\left(u_{i}\right)$, we get hence of path of length 
2 ending with $x_{i} u_{i}$, and we are sure that this path cannot be shorten at the other end, since $F$ is a strong matching. Let $\mathcal{T}^{\prime \prime}$ be the partition so obtained. $\mathcal{T}^{\prime \prime \prime}$ being normal and $\mathcal{T}^{\prime \prime}$ having the same number of trails $\mathcal{T}^{\prime \prime}$ is also normal by Proposition 2.2 .

For each vertex $v \neq u_{i} 1 \leq i \leq k, e_{\mathcal{T}^{\prime \prime \prime}}(v)=e_{\mathcal{T}^{\prime \prime}}(v)$ and we have thus $e_{\mathcal{T}}(v) \neq e_{\mathcal{T}^{\prime}}(v) \neq e_{\mathcal{T}^{\prime \prime}}(v)$. For $v=u_{i} 1 \leq i \leq k$, we have $e_{\mathcal{T}}\left(u_{i}\right)=u_{i} v_{i}$, $e_{\mathcal{T}^{\prime}}\left(u_{i}\right)=u_{i} p\left(u_{i}\right)$ and $e_{\mathcal{T}^{\prime \prime}}\left(u_{i}\right)=u_{i} x_{i}$.

$\mathcal{T}, \mathcal{T}^{\prime}$ and $\mathcal{T}^{\prime \prime}$ are thus compatible , $\mathcal{T}$ is odd, $\mathcal{T}^{\prime}$ has length 3 and $\mathcal{T}^{\prime \prime}$ has length 4 as claimed.

In fact we can extend the result to cubic graphs with multiple edges.

Theorem 4.5 Let $G$ be a 3-edge colourable cubic graph then $G$ has three compatible normal partitions $\mathcal{T}, \mathcal{T}^{\prime}$ and $\mathcal{T}^{\prime \prime}$ such that

- $\mathcal{T}$ is odd

- $\mathcal{T}^{\prime}$ has length 3

- $\mathcal{T}^{\prime \prime}$ has length at most 4

Proof By induction on the number of vertices of $G$. In Figure 5 we can see that the result holds for the cubic graph with two vertices and three edges. If $G$ is simple, we are done by Theorem 4.4. So assume that $G$ has at least 4 vertices and let $u$ and $v$ be two vertices joined by two edges $e_{1}$ and $e_{2}$. Let $x$ be the third vertex adjacent to $u$ and $y$ the one adjacent to $v$. Let $G^{\prime}$ be the graph obtained from $G$ by deleting $u$ and $v$ and adding a new edge $e$ between $x$ and $y$. From the hypothesis of induction, let $\mathcal{Q}, \mathcal{Q}^{\prime}$ and $\mathcal{Q}^{\prime \prime}$ be three compatible normal partitions of $G^{\prime}$. We have to discuss three cases following the fact that $e$ is in $\mathcal{Q}, \mathcal{Q}^{\prime}$ or $\mathcal{Q}^{\prime \prime}$

\section{case 1: $e$ is an internal edge of a trail $Q \in \mathcal{Q}$}

In that case $e$ is an end edge of a trail $Q^{\prime} \in \mathcal{Q}^{\prime}$ as well as an end edge of a trail $Q^{\prime \prime} \in \mathcal{Q}^{\prime \prime}$. Without loss of generality, we assume that $e_{\mathcal{Q}^{\prime}}(x)=x y$ and $e_{\mathcal{Q}^{\prime \prime}}(y)=y x$. Hence $Q^{\prime}$ and $Q^{\prime \prime}$ end both with the edge $x y$. Let $T$ be the trail obtained from $Q$ by deleting the edge $x y$ and adding the path $x u e_{1} v y$ (the notation $u e_{1} v$ means that we use explicitly the edge $e_{1}$ in order to connect $u$ and $v$ ). Let $T^{\prime}$ be the trail obtained from $Q^{\prime}$ by deleting the edge $x y$ and adding the edge $y v$. Let $T^{\prime \prime}$ be the trail obtained from $Q^{\prime \prime}$ by deleting the edge $y x$ and adding the edge $x u$. Then we can construct $\mathcal{T}, \mathcal{T}^{\prime}$ and $\mathcal{T}^{\prime \prime}$ three compatible normal partitions of $G$ in the following way: 
- $\mathcal{T}=\mathcal{Q}-Q+T+u e_{2} v$

- $\mathcal{T}^{\prime}=\mathcal{Q}^{\prime}-Q^{\prime}+T^{\prime}+x u e_{2} v e_{1} u$

- $\mathcal{T}^{\prime \prime}=\mathcal{Q}^{\prime \prime}-Q^{\prime \prime}+T^{\prime \prime}+y v e_{2} u e_{1} v$

We can check that the conditions on the lengths are verified for $\mathcal{T}, \mathcal{T}^{\prime}$ and $\mathcal{T}^{\prime \prime}$.

case 2: $e$ is an internal edge of a trail $Q^{\prime} \in \mathcal{Q}^{\prime}$

In that case $e$ is an end edge of a trail $Q \in \mathcal{Q}$ as well as an end edge of a trail $Q^{\prime \prime} \in \mathcal{Q}^{\prime \prime}$. Without loss of generality, we assume that $e_{\mathcal{Q}}(y)=x y$ and $e_{\mathcal{Q}^{\prime \prime}}(x)=y x$. Hence $Q$ and $Q^{\prime \prime}$ end both with the edge $x y$. Let us recall that $Q^{\prime}$ has length 3. Let $z x$ and $t y$ be the end edges of $Q$. Let $T^{\prime \prime}$ be the trail obtained from $Q^{\prime \prime}$ by deleting the edge $y x$ and adding the edge $y v$. Let $T$ be the trail obtained from $Q$ by deleting the edge $x y$ and adding the path $x u e_{1} v y$

Then we can construct $\mathcal{T}, \mathcal{T}^{\prime}$ and $\mathcal{T}^{\prime \prime}$ three compatible normal partitions of $G$ in the following way:

- $\mathcal{T}=\mathcal{Q}-Q+T+$ xue $_{1} v e_{2} u$

- $\mathcal{T}^{\prime}=\mathcal{Q}^{\prime}-Q^{\prime}+z x u e_{2} v+$ tyve $_{1} u$

- $\mathcal{T}^{\prime \prime}=\mathcal{Q}^{\prime \prime}-Q^{\prime \prime}+T^{\prime \prime}+y v e_{2} u e_{1} v$

We can check that the conditions on the lengths are verified for $\mathcal{T}, \mathcal{T}^{\prime}$ and $\mathcal{T}^{\prime \prime}$.

case 3: $e$ is an internal edge of a trail $Q^{\prime \prime} \in \mathcal{Q}^{\prime \prime}$

A similar technique can be used to solve this case.

Theorem 4.6 Let $G$ be a cubic graph. Then the following statements are equivalent

i) $G$ can be provided with three compatible normal partitions of length 3

ii) $G$ can be provided with three compatible normal odd partitions where each edge is an internal edge in exactly one partition

iii) $G$ is bipartite

Proof Assume first that $G$ can be provided with three compatible normal partitions of length 3 , say $\mathcal{T}, \mathcal{T}^{\prime}$ and $\mathcal{T}^{\prime \prime}$. Since the average length of each partition is 3 (Proposition 2.3), each trail of each partition has length 3. $\mathcal{T}$, $\mathcal{T}^{\prime}$ and $\mathcal{T}^{\prime \prime}$ are thus three normal odd partitions and from Proposition 4.2, each edge is the internal edge of one trail in exactly one partition. Conversely assume that $G$ can be provided with three compatible normal odd partitions where each edge is an internal edge in exactly one partition. Then, by Proposi- 
tion 4.2 there is no trail of length 1 in any of these partitions. Since the average length of each partition is 3 , that means that each trail in each partition has length 3 . Hence $(i) \equiv(i i)$.

We prove now that $(i) \equiv($ iii $)$. Let $\mathcal{T}, \mathcal{T}^{\prime}$ and $\mathcal{T}^{\prime \prime}$ three compatible normal partitions of length 3. Following the proof of Theorem 3.2 the internal edges of trails of $\mathcal{T}\left(\mathcal{T}^{\prime}\right.$ and $\mathcal{T}^{\prime \prime}$ respectively) constitute a perfect matching (say $M$ $M^{\prime}$ and $M^{\prime \prime}$ respectively).

Let $a_{0} a_{1} a_{2} a_{3}$ be a trail of $\mathcal{T}$ and let $b_{1}$ and $b_{2}$ the third neighbors of $a_{1}$ and $a_{2}$ respectively. By definition, we have $e_{\mathcal{T}}\left(a_{1}\right)=a_{1} b_{1}$ and $e_{\mathcal{T}}\left(a_{2}\right)=a_{2} b_{2}$.

Since $a_{0} a_{1}$ and $a_{2} a_{3}$ must be internal edges in a trail of $\mathcal{T}^{\prime}$ or (exclusively) $\mathcal{T}^{\prime \prime}$, without loss of generality we may assume that $a_{0} a_{1}$ is an internal edge of a trail $T_{1}^{\prime}$ of $\mathcal{T}^{\prime}$. $T_{1}^{\prime}$ does not use $a_{1} a_{2}$ otherwise $e_{\mathcal{T}^{\prime}}\left(a_{1}\right)=a_{1} b_{1}$, a contradiction with $e_{\mathcal{T}}\left(a_{1}\right)=a_{1} b_{1}$ since $\mathcal{T}$ and $\mathcal{T}^{\prime}$ are compatible. Hence $T_{1}^{\prime}$ uses $a_{1} b_{1}$ and $e_{\mathcal{T}^{\prime}}\left(a_{1}\right)=a_{1} a_{2}$.

Assume now that $a_{2} a_{3}$ is an internal edge of a trail $T_{2}^{\prime}$ of $\mathcal{T}^{\prime}$. Reasoning in the same way, we get that $e_{\mathcal{T}^{\prime}}\left(a_{2}\right)=a_{2} a_{1}$. These two results leads to the fact that $a_{1} a_{2}$ must be a trail in $\mathcal{T}^{\prime}$, which is impossible since each trail has length 3 .

Hence, whenever $a_{0} a_{1}$ is supposed to be an internal edge in a trail of $\mathcal{T}^{\prime}$, we must have $a_{2} a_{3}$ as an internal edge in a trail of $\mathcal{T}^{\prime \prime}$. The two internal vertices of $a_{0} a_{1} a_{2} a_{3}$ can be thus distinguished, following the fact that the end edge of $\mathcal{T}$ to whom they are incident is internal in $\mathcal{T}^{\prime}$ (say red vertices) or $\mathcal{T}^{\prime \prime}$ (say blue vertices). The same holds for each trail in $\mathcal{T}$ (and incidently for each partition $\mathcal{T}^{\prime}$ and $\left.\mathcal{T}^{\prime \prime}\right)$. The edge $a_{1} b_{1}$ as end-edge of $\mathcal{T}$ cannot be an internal edge in $T^{\prime}$ since the trail of length 3 going through $a_{0} a_{1}$ ends with $a_{1} b_{1}$. Hence $a_{1} b_{1}$ is an internal edge in $\mathcal{T}^{\prime \prime}$ and $b_{1}$ is a blue vertices. Considering now $a_{0}$, this vertex is the internal vertex of a trail of length 3 of $\mathcal{T}$. Since $a_{0} a_{1} \in M^{\prime}$ and $M^{\prime}$ is a perfect matching, $a_{0}$ cannot be incident to an other internal edge of a trail in $\mathcal{T}^{\prime}$ and $a_{0}$ must be a blue vertex. Hence $a_{1}$ is a red vertex and its neighbors are all blue vertices. Since we can perform this reasoning in each vertex, $G$ is bipartite as claimed.

Conversely, assume that $G$ is bipartite and let $V(G)=\{W, B\}$ be the bipartition of its vertex set. In the following, a vertex in $W$ will be represented by a circle (०) while a vertex in $B$ will be represented by a bullet $(\bullet)$. ¿From König's theorem [7] $G$ is a 3 -edge colourable cubic graph . Let us consider a coloring of its edge set with three colors $\{\alpha, \beta, \gamma\}$. Let us denote by $\alpha \bullet \beta \circ \gamma$ a trail of length 3 which is obtained in considering an edge $u v(u \in B$ and $v \in W$ ) colored with $\beta$ together with the edge colored $\alpha$ incident with $u$ and the edge colored with $\gamma$ incident with $v$. It can be easily checked that the set $\mathcal{T}$ of $\alpha \bullet \beta \circ \gamma$ trails of length 3 is a normal odd partition of length 3 . We can 
define in the same way $\mathcal{T}^{\prime}$ as the set of $\beta \bullet \gamma \circ \alpha$ trails of length 3 and $\mathcal{T}^{\prime \prime}$ as the set of $\gamma \bullet \alpha \circ \beta$ trails of length 3 .

Hence $\mathcal{T}, \mathcal{T}^{\prime}$ and $\mathcal{T}^{\prime \prime}$ is a set of three normal odd partitions of length 3 . We claim that these partitions are compatible. Indeed, let $v \in W$ be a vertex and $u_{1}, u_{2}$ and $u_{3}$ its neighbors. Assume that $u_{1} v$ is colored with $\alpha, u_{2} v$ is colored with $\beta$ and $u_{3} v$ is colored with $\gamma$. Hence $u_{1} v$ is internal in a $\gamma \bullet \alpha \circ \beta$ trail of $\mathcal{T}^{\prime \prime}$ and $e_{\mathcal{T}^{\prime \prime}}(v)=v u_{3}$. The edge $u_{2} v$ is internal in a $\alpha \bullet \beta \circ \gamma$ trail of $\mathcal{T}$ and $e_{\mathcal{T}}(v)=v u_{1}$. The edge $u_{3} v$ is internal in a $\beta \bullet \gamma \circ \alpha$ trail of $\mathcal{T}^{\prime}$ and $e_{\mathcal{T}^{\prime}}(v)=v u_{2}$. Since the same reasoning can be performed in each vertex of $G$, the three partitions $\mathcal{T}, \mathcal{T}^{\prime}$ and $\mathcal{T}^{\prime \prime}$ are compatible.

Theorem 4.7 Let $G$ be a cubic graph with three compatible normal partitions $\mathcal{T}, \mathcal{T}^{\prime}$ and $\mathcal{T}^{\prime \prime}$ such that

- $\mathcal{T}$ has length 3

- $\mathcal{T}^{\prime}$ and $\mathcal{T}^{\prime \prime}$ are odd

Then $G$ is a 3-edge colourable cubic graph.

Proof Since $\mathcal{T}$ has length 3, every trail of $\mathcal{T}$ has length 3. Hence there is no edge which can be an internal edge of a trail of $\mathcal{T}^{\prime}$ and a trail of $\mathcal{T}^{\prime \prime}$, since, by Proposition 4.2 such an edge would be a trail of length 1 in $\mathcal{T}$. The perfect matchings associated to $\mathcal{T}^{\prime}$ and $\mathcal{T}^{\prime \prime}$ (see Theorem 3.2) are thus disjoint and induce an even 2-factor of $G$, which means that $G$ is a 3 -edge colourable cubic graph, as claimed.

Proposition 4.8 Let $G$ be a cubic graph which can be provided with three compatible normal odd partitions. Then the graph $G^{\prime}$ obtained by replacing a vertex by a triangle, can also be provided with three compatible normal odd partitions.

Proof Let $u$ be a vertex of $G$ and $v_{1}, v_{2}, v_{3}$ its neighbors (not necessarily distinct). Assume that $\mathcal{T}, \mathcal{T}^{\prime}$ and $\mathcal{T}^{\prime \prime}$ is a set of 3 compatible normal odd partitions of $G$ such that, $e_{\mathcal{T}}(u)=u v_{1}, e_{\mathcal{T}^{\prime}}(u)=u v_{2}$ and $e_{\mathcal{T}^{\prime \prime}}(u)=u v_{3}$. Let $T_{1}$ and $T_{2}$ the two trails of $\mathcal{T}$ such that $u$ is an end of $T_{1}$ and an internal vertex of $T_{2} . T_{1}^{1}$ ending in $v_{1}, T_{1}^{2}$ ending in $v_{2}$ and $T_{2}^{2}$ ending in $v_{3}$ denote the subtrails of $T_{1}$ and $T_{2}$ obtained by deleting $u$. We define similarly $T_{1}^{\prime 1}$ ending in $v_{2}, T_{1}^{\prime 2}$ ending in $v_{1}$ and $T_{2}^{\prime 2}$ ending in $v_{3}$ when considering $T_{1}^{\prime}$ and $T_{2}^{\prime}$ in $\mathcal{T}^{\prime}$ as well as 
$T_{1}^{\prime \prime} 1$ ending in $v_{3}, T_{1}^{\prime \prime 2}$ ending in $v_{2}$ and $T_{2}^{\prime \prime 2}$ ending in $v_{1}$ when considering $T_{1}^{\prime \prime}$ and $T_{2}^{\prime \prime}$ in $\mathcal{T}^{\prime \prime}$.

When we transform $G$ in $G^{\prime}$ the vertex $u$ is deleted and replaced by the triangle $u_{1}, u_{2}, u_{3}$ with $u_{i}$ joined to $v_{i}(i=1,2,3)$.

Let $\mathcal{Q}, \mathcal{Q}^{\prime}$ and $\mathcal{Q}^{\prime \prime}$ be defined in $G^{\prime}$ by

$$
\begin{gathered}
\mathcal{Q}=\mathcal{T}-\left\{T_{1}, T_{2}\right\}+\left\{T_{1}^{1}+v_{1} u_{1}, T_{1}^{2}+v_{2} u_{2} u_{1} u_{3} v_{3}+T_{2}^{2}, u_{2} u_{3}\right\} \\
\mathcal{Q}^{\prime}=\mathcal{T}^{\prime}-\left\{T_{1}^{\prime}, T_{2}^{\prime}\right\}+\left\{T_{1}^{\prime 1}+v_{2} u_{2}, T_{1}^{\prime 2}+v_{1} u_{1} u_{2} u_{3} v_{3}+T_{2}^{\prime 2}, u_{1} u_{3}\right\} \\
\mathcal{Q}^{\prime \prime}=\mathcal{T}^{\prime \prime}-\left\{T_{1}^{\prime \prime}, T_{2}^{\prime \prime}\right\}+\left\{T_{1}^{\prime \prime}+v_{3} u_{3}, T_{1}^{\prime \prime 2}+v_{2} u_{2} u_{1} u_{3} v_{3}+T_{2}^{\prime \prime 2}, u_{2} u_{1}\right\}
\end{gathered}
$$

It is a routine matter to check that $\mathcal{Q}, \mathcal{Q}^{\prime}$ and $\mathcal{Q}^{\prime \prime}$ are three compatible normal odd partitions.

It can be pointed out that cubic graphs with with three compatible normal odd partitions are bridgeless.

Proposition 4.9 Let $G$ be a cubic graph with three compatible normal odd partitions. Then $G$ is bridgeless.

Proof Assume that $x y$ is a bridge of $G$ and let $C$ be the connected component of $G-x y$ containing $x$. Since $G$ has three compatible normal odd partitions, one of these partitions, say $\mathcal{T}$, is such that $e_{\mathcal{T}}(x)=x y$. The edges of $C$ are thus partitioned into odd trails (namely the trace of $\mathcal{T}$ on $C$ ). We have

$$
m=|E(C)|=\frac{3(|C|-1)+2}{2}
$$

and $m$ is even whenever $|C| \equiv 3 \bmod 4$ while $m$ is odd whenever $|C| \equiv 1 \bmod 4$. The trace of $\mathcal{T}$ on $C$ is a set of $\frac{|C|-1}{2}$ trails and this number is odd when $|C| \equiv 3 \bmod 4$ and even otherwise. Hence, when $|C| \equiv 3 \bmod 4$ we must have an odd number of odd trails partitioning $E(C)$ but, in that case $m$ is even and when $|C| \equiv 1 \bmod 4$ we must have an even number of odd trails partitioning $E(C)$ but, in that case $m$ is odd, contradiction.

Fan and Raspaud [3] conjectured that any bridgeless cubic graph can be provided with three perfect matching with empty intersection.

Theorem 4.10 Let $G$ be a cubic graph with three compatible normal odd partitions then there exist 3 perfect matching $M, M^{\prime}$ and $M^{\prime \prime}$ such that $M \cap M^{\prime} \cap$ $M^{\prime \prime}=\emptyset$. 


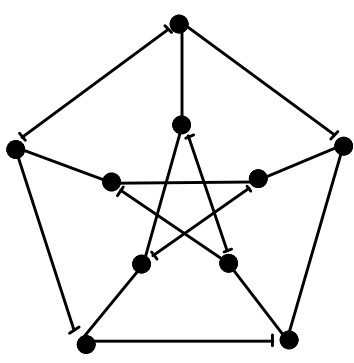

(a)

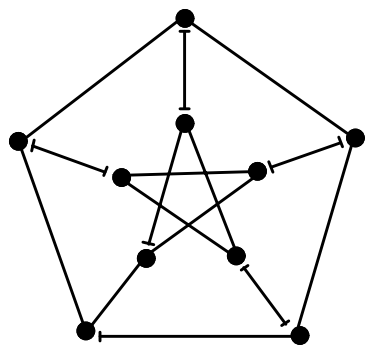

(b)

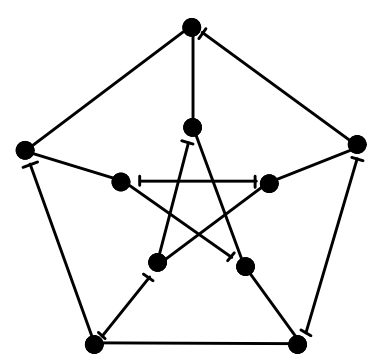

(c)

Fig. 6. Three compatible normal odd partitions of the Petersen's graph

Proof Following the proof of Theorem 3.2 the odd edges of trails of $\mathcal{T}\left(\mathcal{T}^{\prime}\right.$ and $\mathcal{T}^{\prime \prime}$ respectively) constitute a perfect matching (say $M M^{\prime}$ and $M^{\prime \prime}$ respectively). Let $v$ be any vertex and $u_{1}, u_{2}$ and $u_{3}$ its neighbors. $\mathcal{T}, \mathcal{T}^{\prime}$ and $\mathcal{T}^{\prime \prime}$ being compatible, we can suppose that $e_{\mathcal{T}}(v)=v u_{1}, e_{\mathcal{T}^{\prime}}(v)=v u_{2}$ and $e_{\mathcal{T}^{\prime \prime}}(v)=v u_{3}$. Since $v u_{1}$ is an end edge of a trail of $\mathcal{T}$, this edge is not an odd edge relatively to $\mathcal{T}$. That means that $v u_{1} \notin M$. In the same way $v u_{2} \notin M^{\prime}$ and $v u_{3} \notin M^{\prime \prime}$. Hence, any edge incident to $v$ is contained in at most two perfect matchings among $M, M^{\prime}$ and $M^{\prime \prime}$. Which means that $M \cap M^{\prime} \cap M^{\prime \prime}=\emptyset$

Theorem 4.10 above implies that Fan-Raspaud Conjecture is true for graphs with 3 compatible normal odd partitions. By the way, this conjecture seems to be originated independently by Jackson. Goddyn [5] indeed mentioned this problem proposed by Jackson for $r$-graphs $(r$-regular graphs with an even number of vertices such that all odd cuts have size at least $r$, as defined by Seymour [10]) in the proceedings of a joint summer research conference on graphs minors which dates back 1991. It seems difficult to characterize the class of cubic graphs with three compatible normal odd partitions. The Petersen's graph has this property (see Figure 6). In a forthcoming paper we prove that 3-edge colorable graphs also have this property as well as the flower snarks.

\section{References}

[1] J. A. Bondy. Basic graph theory: Paths and circuits. In M. Grötschel R.L. Graham and L. Lovász, editors, Handbook of Combinatorics, volume 1, pages 3-112. Elsevier, North-Holland, 1995.

[2] A. Bouchet and J.L. Fouquet. Trois types de dcompositions d'un graphe chaînes. Annals of Discrete Mathematics, 17:131-141, 1983. 
[3] G. Fan and A. Raspaud. Fulkerson's conjecture and circuit covers. J. Comb. Theory Ser. B, $\underline{6} 1: 133-138,1994$.

[4] J.L. Fouquet, H. Thuillier, J.M. Vanherpe, and A.P. Wojda. On odd and semiodd linear partitions of cubic graphs. to appear.

[5] L. Goddyn. Cones, lattices and Hilbert base of circuits and perfect matching. In N. Robertson and P. Seymour, editors, Graph Structure Theory, Contemporary Mathematics Volume 147, pages 419-439. American Mathematical Society, 1993.

[6] R. Halin. A theorem on n-connected graphs. J. Combin. Theory, pages 150-154, 1969.

[7] D. König. Über Graphen und ihre Anwendung auf Determinantentheorie und Mengenlehre. Math.Ann, 77:453-465, 1916.

[8] A. Kotzig. Moves without forbidden transitions. Mat.-Fyz. Časopis, 18:76-80, 1968. MR 39\#4038.

[9] H. Li. Perfect path double covers in every simple graphs. J. Graph. Theory, 14:645-650, 1990. MR 91h\#05052.

[10] P. Seymour. On multi-colourings of cubic graphs, and conjectures of Fulkerson and Tutte. Proc. London Math. Soc.(3), 38:423-460, 1979. 